\title{
On Some Classes with Norms of Meromorphic Function Spaces Defined by General Spherical Derivatives
}

\author{
A. El-Sayed Ahmed ${ }^{1}{ }^{1}$ and S. Attia Ahmed ${ }^{2,3}$ \\ ${ }^{1}$ Mathematics Department, Faculty of Science, Taif University, P. O. Box 11099, Taif 21944, Saudi Arabia \\ ${ }^{2}$ Mathematics Department, Faculty of Science, Assiut University, Assiut, Egypt \\ ${ }^{3}$ Umm Al Qura University, AL-Qunfudah University College, Makka, Saudi Arabia
}

Correspondence should be addressed to A. El-Sayed Ahmed; ahsayed80@hotmail.com

Received 10 February 2021; Revised 1 March 2021; Accepted 28 March 2021; Published 14 April 2021

Academic Editor: Zakia Hammouch

Copyright (c) $2021 \mathrm{~A}$. El-Sayed Ahmed and S. Attia Ahmed. This is an open access article distributed under the Creative Commons Attribution License, which permits unrestricted use, distribution, and reproduction in any medium, provided the original work is properly cited.

\begin{abstract}
The main concerned target of this article is to define and study some concerned classes of meromorphic function spaces using the general spherical derivatives. The general Besov-type classes of meromorphic functions as well as the general normal functions are considered intensively and both are compared deeply with each other. Specifically, multiple results concerning general meromorphic-type classes as well as non-normal classes are obtained by the help of general spherical derivatives. The concerned results are proved by constructing some specific mild conditions on the sequences of points belonging to the concerned meromorphic-type classes. The obtained results generalize and improve the corresponding previous results in some concerned respects. The concerned proofs and methods are simply presented.
\end{abstract}

\section{Introduction}

The area of complex function spaces is fundamental and essential in many branches of pure and applied mathematics. Some decades ago, there have been obvious interests on meromorphic function classes, from concerned point of view of their singularities. For various studies on meromorphic function spaces, we may refer to all citations therein. As a concerned result, some new general classes of meromorphic functions shall be introduced by using the general spherical derivatives, which will be associated to obtain the new classes of meromorphic function spaces. Fundamental concerned properties of these concerned aforementioned meromorphic-type classes which include generalizations of meromorphic Besov spaces as well as normal function classes shall be studied and intensively discussed. As a concerned consequence of our investigation, some relevant special cases can be pointed out. Furthermore, to capture some new generalized results under the current concerned proofs, some new concepts and definitions are introduced. Let $U=$ $\{w:|w|<1\}$ be the open unit disk in the complex plane $\mathbb{C}$ and let $\mathrm{d} m(w)$ be the usual Euclidean area element on $U$. The symbol $M(U)$ stands for the concerned class of all meromorphic functions in $U$. The pseudohyperbolic metric between the points $w$ and $z$ is defined by $\mathrm{d}(z, w)=\left|\varphi_{z}(w)\right|$. For $0<R<1$, assume that $U(a, R)=\{w \in U: d(w, a)<r\}$ defines the concerned pseudohyperbolic disc which is centered $a \in U$ with the specific radius $R$. For $0<q<\infty$ and $0<s<\infty$, the classes $M^{\#}(p, q, s)$ are defined by (see [1] pp.10)

$$
M^{\#}(p, q, s)=\left\{h \in M(U): \sup _{a \in U} \iint_{U}\left(h^{\#}(w)\right)^{p}\left(1-|w|^{2}\right)^{q}\left(1-\left|\varphi_{a}(w)\right|^{2}\right)^{s} \mathrm{~d} m(w)<\infty\right\},
$$


where $h^{\#}(w)=\left(|h \prime(w)| /\left(1+|h(w)|^{2}\right)\right)$ is the usual spherical derivative of $h$. The meromorphic $M^{\#}(q, q-2,0)$ classes are called the meromorphic Besov classes and denoted by $B_{q}^{\#}$, for which

$$
B_{q}^{\#}=\left\{h \in M(U): \sup _{a \in U} \iint_{U}\left(h^{\#}(w)\right)^{q}\left(1-|w|^{2}\right)^{q-2} \mathrm{~d} m(w)<\infty\right\}
$$

For the analytic corresponding classes of Besov spaces, we cite [2-7]. In this article, the general meromorphic Besov-type classes always refer to the concerned classes $B^{\#}(q, q-2, s ; n)$. Using the general spherical derivative $\left(\left|h^{(n)}(w)\right| /\left(1+|h(w)|^{n+1}\right)\right)$ (see [8]), we give the following general meromorphic spaces.

Let $n \in \mathbb{N}, 0<q<\infty, 0<s<\infty$. Then, the general meromorphic Besov-type spaces are defined by

$$
B^{\#}(q, q-2, s ; n)=\left\{h \in M(U): \sup _{a \in U} \int_{U}\left[\frac{\left|h^{(n)}(w)\right|}{1+|h(w)|^{n+1}}\right]^{q}\left(1-|w|^{2}\right)^{q-2}\left(1-\left|\varphi_{a}(w)\right|^{2}\right)^{s} \mathrm{~d} m(w)<\infty\right\},
$$

where the concerned weight function is $\left(1-|w|^{2}\right)^{q-2}$ $\left(1-\left|\varphi_{a}(w)\right|^{2}\right)^{s}$ and $w \in U$. Here, $\varphi_{a}(w)$ denotes the usual Möbius transformation $\varphi_{a}(w)=((a-w) /(1-\bar{a} w))$. Also, $h^{(n)}(w)=\frac{\mathrm{d}^{n} h(w)}{\mathrm{d} w^{n}}$, that is, we have " $n$ " times derivatives, $n \in \mathbb{N}$.

The concerned meromorphic counterpart of the Blochtype space is the class of all concerned normal functions $\mathcal{N}$ (see $[1,9])$; this class of meromorphic functions can be extended to the following concerned class.

Definition 1. Assume that $h$ is a meromorphic function in $U$. When

$$
\|h\|_{\mathcal{N}_{n}}=\sup _{w \in U}\left(1-|w|^{2}\right) \frac{\left|h^{(n)}(w)\right|}{1+|h(w)|^{n+1}}<\infty,
$$

$h \in \mathcal{N}_{n}$ of concerned normal functions.
Definition 2. Suppose that the function $h$ stands for a concerned meromorphic function in $U$. The concerned sequence of points $\left\{a_{m}\right\}\left(\left|a_{m}\right| \longrightarrow 1\right)$ in $U$ is called a $q_{(N, n)}$-sequence if

$$
\lim _{m \rightarrow \infty} \frac{\left|h^{(n)}(w)\right|}{1+|h(w)|^{n+1}}\left(1-\left|a_{m}\right|^{2}\right)=+\infty .
$$

In Definitions 2 , by letting $n=1$, we obtain the class of all usual normal functions $\mathcal{N}$ (see $[1,9]$ ). For more interesting various studies on different meromorphic function classes, we refer to [10-15] and others. The following definitions can be introduced.

Definition 3. Assume that $h$ is a meromorphic function in $U$. For $2<q<\infty$ and $0<s<\infty$, the concerned sequence of points $\left\{a_{m}\right\}\left(\left|a_{m}\right| \longrightarrow 1\right)$ in $U$ is called a $b_{(q, n)}$-sequence if

$$
\lim _{m \longrightarrow \infty} \iint_{U}\left[\frac{\left|h^{(n)}(w)\right|}{1+|h(w)|^{n+1}}\right]^{q}\left(1-|w|^{2}\right)^{q-2}\left(1-\left|\varphi_{a_{m}}(w)\right|^{2}\right)^{s} \mathrm{~d} m(w)=+\infty
$$

\section{Families of $\mathbf{b}_{(q, n)}$ - and $\mathbf{q}_{(N, n)}$-Type Sequences}

Theorem 1. Let $h \in M(U)$. Suppose that $\left\{a_{m}\right\}$ defines the $q_{(N, n)}$ type sequence, thus any sequence of points $\left\{c_{m}\right\}$ in $U$, such that $d\left(a_{m}, c_{m}\right) \longrightarrow 0$ is a $b_{(q, n)}$-type sequence for all values of $q$ with $2<q<\infty$.
Proof. In view of [16], we can find two concerned sequences $\left\{c_{m}\right\} \subset U$ and $\left\{d_{m}\right\} \subset \mathbb{R}^{+}$, with $\mathrm{d}\left(a_{m}, c_{m}\right) \longrightarrow 0$ with

$$
\frac{d_{m}}{\left(1-\left|c_{m}\right|^{2}\right)} \longrightarrow 0
$$

where the concerned sequence of functions $\left\{h_{m}(t)\right\}=\left\{h\left(b_{m}+d_{m} t\right)\right\}$ has uniformly converging type on 
each concerned compact subset of $\mathbb{C}$ to a concerned nonconstant meromorphic function $G(t)$. Thus,

$$
\begin{aligned}
& \sup _{c_{m} \in U} \iint_{U}\left[\frac{\left|h^{(n)}(w)\right|}{1+|h(w)|^{n+1}}\right]^{q}\left(1-|w|^{2}\right)^{q-2}\left(1-\left|\varphi_{c_{m}}(w)\right|^{2}\right)^{s} \mathrm{~d} m(w) \\
& \quad \geq \iint_{U\left(c_{m},(1 / e)\right)}\left[\frac{\left|h^{(n)}(w)\right|}{1+|h(w)|^{n+1}}\right]^{q}\left(1-|w|^{2}\right)^{q-2}\left(1-\left|\varphi_{c_{m}}(w)\right|^{2}\right)^{s} \mathrm{~d} m(w) \\
& \quad \geq \iint_{U(0, r)}\left[\frac{\left|h_{m}^{(n)}(t)\right|}{1+\left|h_{m}(t)\right|^{n+1}}\right]^{q}\left(1-\left|c_{m}+d_{m} t\right|^{2}\right)^{q-2}\left(1-\left|\varphi_{c_{m}}\left(c_{m}+\mathrm{d}_{m} t\right)\right|^{2}\right)^{s} \mathrm{~d}_{m}^{2-q} \mathrm{~d} m(t) \\
& \quad=\iint_{U(0, r)}\left[\frac{\left|h_{m}^{(n)}(t)\right|}{1+\left|h_{m}(t)\right|^{n+1}}\right]^{q}\left(\frac{1-\left|c_{m}+d_{m} t\right|^{2}}{d_{m}}\right)^{q-2} \times\left(\left|\frac{c_{m}-\left(c_{m}+d_{m} t\right)}{1-\bar{c}_{m}\left(c_{m}+d_{m} t\right)}\right|^{2}\right)^{s} \mathrm{~d} m(t) \\
& \quad=\iint_{U(0, r)}\left[\frac{\left|h_{m}^{(n)}(t)\right|}{1+\left|h_{m}(t)\right|^{n+1}}\right]^{q}\left(\frac{1-\left|c_{m}+d_{m} t\right|^{2}}{d_{m}}\right)^{q-2} \times\left(1-\left|\frac{1}{\left(\left(1-\left|c_{m}\right|^{2}\right) / \mathrm{d}_{m} t\right)-\bar{c}_{m}}\right|^{2}\right)^{s} \mathrm{~d} m(t) .
\end{aligned}
$$
that

Using the uniform convergence techniques, we deduce

where the last defined integral is positive, since $G(t)$ is a concerned nonconstant meromorphic function. Further, by (6), when $m \longrightarrow \infty$, we conclude that

$$
\iint_{U(0, r)}\left[\frac{\left|h_{m}^{(n)}(t)\right|}{1+\left|h_{m}(t)\right|^{n+1}}\right]^{q} \mathrm{~d} m(t) \longrightarrow \iint_{U(0, r)}\left(G_{n}^{\#}(t)\right)^{q} \mathrm{~d} m(t)
$$

$$
1-\left|\frac{1}{\left(\left(1-\left|c_{m}\right|^{2}\right) / d_{m} t\right)-\bar{c}_{m}}\right|^{2} \longrightarrow 1 \text {. }
$$

Therefore, we can obtain that

$$
\iint_{U(0, r)}\left[\frac{\left|h_{m}^{(n)}(t)\right|}{1+\left|h_{m}(t)\right|^{n+1}}\right]^{q}\left(\frac{1-\left|a_{m}+\mathrm{d}_{m} t\right|^{2}}{\mathrm{~d}_{m}}\right)^{q-2}\left(1-\left|\frac{1}{\left(\left(1-\left|a_{m}\right|^{2}\right) / \mathrm{d}_{m} t\right)-\bar{a}_{m}}\right|^{2}\right)^{s} \mathrm{~d} m(t) \longrightarrow \infty .
$$

Hence, when $2<q<\infty$, we have that

$$
\iint_{U}\left[\frac{\left|h^{(n)}(w)\right|}{1+|h(w)|^{n+1}}\right]^{q}\left(1-|w|^{2}\right)^{q-2}\left(1-\left|\varphi_{c_{m}}(w)\right|^{2}\right)^{s} \mathrm{~d} m(w) \longrightarrow \infty,
$$

Thus, $\left\{c_{m}\right\} \in U$ is a $b_{(q, n)}$-type sequence for all $q$, with $2<q<\infty$. The proof of Theorem 1 is completely finished.

Theorem 2. We can find a concerned non-normal function $h$ and a concerned sequence $\left\{a_{m}\right\}$ in $U$ which is $a b_{(q, n)}$-sequence for all $q$, with $2<q<\infty$, whereas $\left\{a_{m}\right\}$ is not a $q_{(N, n)}$-sequence.

Proof. Assume that the function $h(w)=\exp (i /(1-w))$ is a non-normal function where $i=\sqrt{-1}$. Considering the concerned sequence $\left\{c_{m}\right\}=\left\{m^{2} /\left(1+m^{2}\right)\right\}$, after simple computation, we deduce that

$$
\lim _{m \rightarrow \infty}\left(1-\left|c_{m}\right|^{2}\right) \frac{\left|h^{(n)}\left(c_{m}\right)\right|}{1+\left|h\left(c_{m}\right)\right|^{n+1}}=+\infty .
$$

Applying Theorem 1 for any concerned sequence of specific points $\left\{a_{m}\right\}$ in $U$, with $\mathrm{d}\left(a_{m}, c_{m}\right) \longrightarrow 0$, we get

$$
\begin{gathered}
\lim _{m \rightarrow \infty} \iint_{U}\left[\frac{\left|h^{(n)}(w)\right|}{1+|h(w)|^{n+1}}\right]^{q}\left(1-|w|^{2}\right)^{q-2} \\
\cdot\left(1-\left|\varphi_{a_{m}}(w)\right|^{2}\right)^{s} \mathrm{~d} m(w)=+\infty
\end{gathered}
$$

for all $q$, with $2<q<\infty$. Let $\left\{a_{m}\right\}=\left\{\left(m^{2} /\left(1+m^{2}\right)\right)-\right.$ $\left.\left(i /\left(m+m^{3}\right)\right)\right\}$, and note that $\mathrm{d}\left(a_{m}, c_{m}\right) \longrightarrow 0$. But

$$
\lim _{m \rightarrow \infty}\left(1-\left|a_{m}\right|^{2}\right) \frac{\left|h^{(n)}\left(a_{m}\right)\right|}{1+\left|h\left(m_{m}\right)\right|^{n+1}}=0 .
$$

Hence, the concerned sequence $\left\{a_{m}\right\}$ is our needed sequence of points. 
Theorem 3. Let $h \in M(U)$ and suppose that $2<q_{1}<q<\infty$ and $0<s_{1}<s<\infty$. For a concerned sequence of points $\left\{a_{m}\right\}$ in the disc $U$, when

$$
\begin{gathered}
\lim _{m \rightarrow \infty} \iint_{U}\left[\frac{\left|h^{(n)}(w)\right|}{1+|h(w)|^{n+1}}\right]^{q}\left(1-|w|^{2}\right)^{q-2}\left(1-\left|\varphi_{a_{m}}(w)\right|^{2}\right)^{s} \mathrm{~d} m(w)=+\infty, \\
\lim _{m \rightarrow \infty} \iint_{U}\left[\frac{\left|h^{(n)}(w)\right|}{1+|h(w)|^{n+1}}\right]^{q_{1}}\left(1-|w|^{2}\right)^{q_{1}-2}\left(1-\left|\varphi_{a_{m}}(w)\right|^{2}\right)^{s_{1}} \mathrm{~d} m(w)=+\infty .
\end{gathered}
$$

Proof. When condition (17) holds, then for $2<q_{1}<q<\infty$ and $0<s_{1}<s<\infty$, using the known inequality of Hölder, we conclude that

$$
\begin{aligned}
& \iint_{U}\left[\frac{\left|h^{(n)}(w)\right|}{1+|h(w)|^{n+1}}\right]^{q_{1}}\left(1-|w|^{2}\right)^{q_{1}-2}\left(1-\left|\varphi_{a_{m}}(w)\right|^{2}\right)^{s_{1}} \mathrm{~d} m(w) \\
& \leq\left(\iint_{U}\left[\frac{\left|h^{(n)}(w)\right|}{1+|h(w)|^{n+1}}\right]^{q}\left(1-|w|^{2}\right)^{q-2}\left(1-\left|\varphi_{a_{m}}(w)\right|^{2}\right)^{s} \mathrm{~d} m(w)\right)^{\left(q_{1} / q\right)} \\
& \quad \times\left(\iint_{U}\left(1-\left|\varphi_{a_{m}}(w)\right|^{2}\right)^{\left(s_{1}-\left(s q_{1} / q\right)\right)\left(q /\left(q-q_{1}\right)\right)}\left(1-|w|^{2}\right)^{-2} \mathrm{~d} m(w)\right)^{\left(1-\left(q_{1} / q\right)\right)} \\
& =\left(\iint_{U}\left[\frac{\left|h^{(n)}(w)\right|}{1+|h(w)|^{n+1}}\right]^{q}\left(1-|w|^{2}\right)^{q-2}\left(1-\left|\varphi_{a_{m}}(w)\right|^{2}\right)^{s} \mathrm{~d} m(w)\right)^{\left(q_{1} / q\right)} \\
& \quad \times\left(\iint_{U}\left(1-|w|^{2}\right)^{\left(\left(s_{1} q-s q_{1}\right) /\left(q-q_{1}\right)-2\right)} \mathrm{d} m(w)\right)^{\left(1-\left(q_{1} / q\right)\right)} .
\end{aligned}
$$

It is obvious to see that $\left(\left(s_{1} q-s q_{1}\right) /\left(q-q_{1}\right)-2\right)=$ $(\eta-2)>-1$, for $\eta>1$, and we obtain

$$
\iint_{U}\left(1-|w|^{2}\right)^{\left(\left(s_{1} q-s q_{1}\right) /\left(q-q_{1}\right)-2\right)} \mathrm{d} m(w)=\iint_{U}\left(1-|w|^{2}\right)^{(\eta-2)} \mathrm{d} m(w)<C_{1}<\infty,
$$

where $\quad C_{1}>0 . \quad$ Therefore, $\quad B^{\#}(q, q-2, s ; n) \subset \quad$ Thus, the following inequality can be followed: $B^{\#}\left(q_{1}, q_{1}-2, s_{1} ; n\right)$.

$$
\begin{aligned}
& \iint_{U}\left[\frac{\left|h^{(n)}(w)\right|}{1+|h(w)|^{n+1}}\right]^{q_{1}}\left(1-|w|^{2}\right)^{q_{1}-2}\left(1-\left|\varphi_{a_{m}}(w)\right|^{2}\right)^{s_{1}} \mathrm{~d} m(w) \\
& \quad \geq \iint_{U}\left[\frac{\left|h^{(n)}(w)\right|}{1+|h(w)|^{n+1}}\right]^{q}\left(1-|w|^{2}\right)^{q-2}\left(1-\left|\varphi_{a_{m}}(w)\right|^{2}\right)^{s} \mathrm{~d} m(w)=+\infty .
\end{aligned}
$$


Then, condition (18) must be verified. Thus, the proof is established completely.

Remark 1. Using the specific condition (17), we deduce that the function $h$ not in the classes $B^{\#}(q, q-2, s ; n)$, this because the concerned meromorphic classes $B^{\#}(q, q-2, s ; n)$ have a specific nesting property and the meromorphic function $h$ is not belong to the meromorphic classes
$B^{\#}\left(q_{1}, q_{1}-2, s_{1}\right)$ when $2<q_{1}<q<\infty$ and $0<s_{1}<s<\infty$. Nevertheless, Theorem 3 shows further details on this case which clearing that the similar concerned sequence of points $\left\{a_{m}\right\}$, for which $B^{\#}(q, q-2, s ; n)$-condition can be excluded, also it excludes the $B^{\#}\left(q_{1}, q_{1}-2, s_{1} ; n\right)$-condition.

Remark 2. From the concerned proof of Theorem 3, we can clearly show that for a fixed $\rho_{0}, 0<\rho_{0}<1$ and $\rho>0$, when

$$
\lim _{m \rightarrow \infty} \iint_{U\left(a_{m}, \rho_{0}\right)}\left[\frac{\left|h^{(n)}(w)\right|}{1+|h(w)|^{n+1}}\right]^{q}\left(1-|w|^{2}\right)^{q-2}\left(1-\left|\varphi_{a_{m}}(w)\right|^{2}\right)^{s} \mathrm{~d} m(w)=+\infty
$$

Thus, we can find a concerned sequence of points $\left\{c_{m}\right\}$ in $\Delta_{\rho}^{n}=\left\{w:\left(1-\left|\varphi_{a}(w)\right|^{2}\right)>\rho\right\}$, for which

$$
\lim _{m \rightarrow \infty}\left(1-\left|c_{m}\right|^{2}\right) \frac{\left|h^{(n)}\left(c_{m}\right)\right|}{1+\left|h\left(c_{m}\right)\right|^{n+1}}=+\infty
$$

Theorem 4. Let $h \in M(U)$. For a concerned sequence of points $\left\{a_{m}\right\} \subset U$, when

$$
\lim _{m \rightarrow \infty}\left(1-\left|a_{m}\right|^{2}\right) \frac{\left|h^{(n)}\left(a_{m}\right)\right|}{1+\left|h\left(a_{m}\right)\right|^{n+1}}=+\infty
$$

for the same concerned sequence $\left\{a_{m}\right\}$, we have

$$
\lim _{m \rightarrow \infty} \iint_{U\left(a_{m}, \rho\right)}\left[\frac{\left|h^{(n)}(w)\right|}{1+|h(w)|^{n+1}}\right]^{q}\left(1-|w|^{2}\right)^{q-2}\left(1-\left|\varphi_{a_{m}}(w)\right|^{2}\right)^{s} \mathrm{~d} m(w)=+\infty
$$

for all values of $q$, $s$ where $2<q<\infty$ and $0<s<\infty$ as well as $\rho$, with $0<\rho<1$.
Proof. Assume that condition (25) holds. Then, we have $\rho_{0}$, $0<\rho_{0}<1$, such that

$$
\lim _{m \rightarrow \infty} \sup \iint_{U\left(a_{m}, \rho_{0}\right)}\left[\frac{\left|h^{(n)}(w)\right|}{1+|h(w)|^{n+1}}\right]^{q}\left(1-|w|^{2}\right)^{q-2}\left(1-\left|\varphi_{a_{m}}(w)\right|^{2}\right)^{s} \mathrm{~d} m(w)=K<+\infty
$$

Thus, we can find a concerned subsequence $\left\{a_{m_{k}}\right\}$ of $\left\{a_{m}\right\}$, for which

$$
\iint_{U\left(a_{m_{k}}, \rho_{0}\right)}\left[\frac{\left|h^{(n)}(w)\right|}{1+|h(w)|^{n+1}}\right]^{q}\left(1-|w|^{2}\right)^{q-2}\left(1-\left|\varphi_{a_{m_{k}}}(w)\right|^{2}\right)^{s} \mathrm{~d} m(w) \leq K+1
$$

this can be verfied for sufficiently large $k$. Let $\rho_{1}, 0<\rho_{1}<\rho_{0}$, $U\left(a_{m_{k}}, \rho_{1}\right)=\left\{w \in U:\left|\varphi_{a_{m_{k}}}(w)\right|<\rho_{1}\right\}$, which verifies that

$$
\frac{K+1}{\left(1-\rho^{2}\right)^{s+q-2}}<\frac{\pi}{2}
$$

This implies that

$$
\iint_{U\left(a_{m_{k}}, \rho_{1}\right)}\left[\frac{\left|h^{(n)}(w)\right|}{1+|h(w)|^{n+1}}\right]^{q} \mathrm{~d} m(w) \leq \frac{K+1}{\left(1-\rho_{1}^{2}\right)^{s+q-2}}<\frac{\pi}{2}
$$

where $\left(1-\left|\varphi_{a_{m_{k}}}(w)\right|^{2}\right) \geq\left(1-\rho_{1}^{2}\right)$. Applying the theorem of Dufresngy (see [15]), we deduce that

$$
\left(1-\left|a_{m_{k}}\right|^{2}\right) \frac{\left|h^{(n)}\left(a_{m_{k}}\right)\right|}{1+\left|h\left(a_{m_{k}}\right)\right|^{n+1}} \leq \frac{1}{\rho_{1}}
$$

and this is a contradiction of the concerned assumption. Therefore, the concerned proof of Theorem 4 is finished.

Theorem 5. Suppose that $h \in M(U)$. For $q, s \in(0, \infty)$, we can find a concerned sequence of points $\left\{a_{m}\right\} \subset U$, for which 


$$
\lim _{m \rightarrow \infty} \iint_{U}\left[\frac{\left|h^{(n)}(w)\right|}{1+|h(w)|^{n+1}}\right]^{q}\left(1-|w|^{2}\right)^{q-2}\left(1-\left|\varphi_{a_{m}}(w)\right|^{2}\right)^{s} \mathrm{~d} m(w)=+\infty
$$

Hence, for any concerned sequence of points $\left\{c_{m}\right\}$ in $U$ such that $\mathrm{d}\left(a_{m}, c_{m}\right) \longrightarrow 0$, we have

$$
\lim _{m \rightarrow \infty} \iint_{U}\left[\frac{\left|h^{(n)}(w)\right|}{1+|h(w)|^{n+1}}\right]^{q}\left(1-|w|^{2}\right)^{q-2}\left(1-\left|\varphi_{c_{m}}(w)\right|^{2}\right) \mathrm{d} m(w)=+\infty
$$

Proof. Let $K_{1}$ and $K_{2}$ be two specific positive constants satisfying $K_{2}<K_{1}$. Suppose that

$$
\begin{aligned}
U_{K_{1}}^{m} & =\left\{w:\left(1-\left|\varphi_{a_{m}}(w)\right|^{2}\right)>K_{1}\right\} \text { and } \\
U_{K_{2}}^{m} & =\left\{w:\left(1-\left|\varphi_{a_{m}}(w)\right|^{2}\right)>K_{2}\right\} .
\end{aligned}
$$

$$
\begin{aligned}
& \iint_{U \backslash U_{M_{2}}^{m}}\left[\frac{\left|h^{(n)}(w)\right|}{1+|h(w)|^{n+1}}\right]^{q}\left(1-|w|^{2}\right)^{q-2}\left(1-\left|\varphi_{c_{m}}(z)\right|^{2}\right)^{s} \mathrm{~d} m(w) \\
& \quad \geq C^{s} \iint_{U \backslash U_{K_{2}}^{m}}\left[\frac{\left|h^{(n)}(w)\right|}{1+|h(w)|^{n+1}}\right]^{q}\left(1-|w|^{2}\right)^{q-2}\left(1-\left|\varphi_{a_{m}}(w)\right|^{2}\right)^{s} \mathrm{~d} m(w) .
\end{aligned}
$$

This inequality holds for any concerned sequence of points $\left\{c_{m}\right\}$ in $U$ with $\mathrm{d}\left(a_{m}, c_{m}\right) \longrightarrow 0$. When

$$
\lim _{m \longrightarrow \infty} \sup \iint_{U \backslash U_{K_{2}}^{m}}\left[\frac{\left|h^{(n)}(w)\right|}{1+|h(w)|^{n+1}}\right]^{q}\left(1-|w|^{2}\right)^{q-2}\left(1-\left|\varphi_{a_{m}}(w)\right|^{2}\right)^{s} \mathrm{~d} m(w)=+\infty .
$$

Therefore, using (10), we obtain

$$
\lim _{m \rightarrow \infty} \sup \iint_{U \backslash U_{K_{2}}^{m}}\left[\frac{\left|h^{(n)}(w)\right|}{1+|h(w)|^{n+1}}\right]^{q}\left(1-|w|^{2}\right)^{q-2}\left(1-\left|\varphi_{c_{m}}(w)\right|^{2}\right)^{s} \mathrm{~d} m(w)=+\infty
$$

If

$$
\lim _{m \longrightarrow \infty} \sup \iint_{U_{K_{2}}^{m}}\left[\frac{\left|h^{(n)}(w)\right|}{1+|h(w)|^{n+1}}\right]^{q}\left(1-|w|^{2}\right)^{q-2}\left(1-\left|\varphi_{a_{m}}(w)\right|^{2}\right)^{s} \mathrm{~d} m(w)=+\infty
$$

Thus, we can consider two cases. 
Case 1. In this case, we can find a concerned sequence of points $\left\{c_{m}\right\}$ in $U_{K_{2}}^{m}$, such that $\mathrm{d}\left(a_{m}, c_{m}\right) \longrightarrow 0$, for which

$$
\lim _{m \rightarrow \infty}\left(1-\left|c_{m}\right|^{2}\right) \frac{\left|h^{(n)}\left(c_{m}\right)\right|}{1+\left|h\left(c_{m}\right)\right|^{n+1}}=+\infty
$$

or we can consider the following case.
Case 2. We can find $R_{0}, 0<R_{0}<e^{-K_{2}}$; also, there exists $\lambda>0$, for which

$$
\left(1-|w|^{2}\right) \frac{\left|h^{(n)}(w)\right|}{1+|h(w)|^{n+1}} \leq \lambda,
$$

where we consider all $w \in U\left(a_{m}, R_{0}\right)$. If Case 1 is verified, by Theorem 1 , for the aforementioned sequence $\left\{c_{m}\right\}$ such that $\mathrm{d}\left(a_{m}, c_{m}\right) \longrightarrow 0$, we deduce that

$$
\lim _{m \rightarrow \infty} \sup \iint_{U}\left[\frac{\left|h^{(n)}(w)\right|}{1+|h(w)|^{n+1}}\right]^{q}\left(1-|w|^{2}\right)^{q-2}\left(1-\left|\varphi_{c_{m}}(w)\right|^{2}\right)^{s} \mathrm{~d} m(w)=+\infty
$$

This is because $\mathrm{d}\left(a_{m}, c_{m}\right) \longrightarrow 0$. Also, when Case 2 holds, using the same concerned conclusions for the concerned weight functions, we obtain that necessarily condition for any concerned sequence of points $\left\{c_{n}\right\}$ such that $\mathrm{d}\left(a_{m}, c_{m}\right) \longrightarrow 0$,

$$
\lim _{m \longrightarrow \infty} \sup \iint_{U}\left[\frac{\left|h^{(n)}(w)\right|}{1+|h(w)|^{n+1}}\right]^{q}\left(1-|w|^{2}\right)^{q-2}\left(1-\left|\varphi_{c_{m}}(w)\right|^{2}\right)^{s} \mathrm{~d} m(w)=+\infty
$$

This is the end of the concerned proof.

Now we are dealing with the following interesting

Assume that $2<q<\infty$ for any concerned sequence $\left\{a_{m}\right\}$ question:

$$
\lim _{m \longrightarrow \infty} \iint_{U}\left[\frac{\left|h^{(n)}(w)\right|}{1+|h(w)|^{n+1}}\right]^{q}\left(1-|w|^{2}\right)^{q-2}\left(1-\left|\varphi_{a_{m}}(w)\right|^{2}\right)^{s} \mathrm{~d} m(w)=+\infty
$$

Is the following equation correct?

$$
\lim _{m \longrightarrow \infty} \sup \iint_{U}\left[\frac{\left|h^{(n)}(w)\right|}{1+|h(w)|^{n+1}}\right]^{q_{1}}\left(1-|w|^{2}\right)^{q_{1}-2}\left(1-\left|\varphi_{a_{m}}(w)\right|^{2}\right)^{s} \mathrm{~d} m(w)=+\infty
$$

for $q_{1}$ with $q<q_{1}$.

We give the answer of this important question by introducing Theorem 6 with its concerned proof.
Definition 4. Let $\left\{a_{m}\right\}$ be any concerned sequence of points in $U$; then, $\left\{a_{m}\right\}$ is said to be a $m_{(q, n)}$-sequence, when

$$
\lim _{m \rightarrow \infty} \sup \iint_{U}\left[\frac{\left|h^{(n)}(w)\right|}{1+|h(w)|^{n+1}}\right]^{q}\left(1-\left|\varphi_{a_{m}}(w)\right|^{2}\right)^{q} \mathrm{~d} m(w)=+\infty
$$

Theorem 6. Let $q \in(2, \infty)$ and assume that

$$
\lim _{m \rightarrow \infty} \iint_{U}\left[\frac{\left|h^{(n)}(w)\right|}{1+|h(w)|^{n+1}}\right]^{q}\left(1-|w|^{2}\right)^{q-2}\left(1-\left|\varphi_{a_{m}}(w)\right|^{2}\right)^{s} \mathrm{~d} m(w)=+\infty
$$


When the concerned sequence of points $\left\{a_{m}\right\}$ in $U$ is not a concerned $m_{(q, n)}$-sequence, for any $q_{1}$ with $q<q_{1}$, we conclude that

$$
\lim _{m \longrightarrow \infty} \iint_{U}\left[\frac{\left|h^{(n)}(w)\right|}{1+|h(w)|^{n+1}}\right]^{q_{1}}\left(1-|w|^{2}\right)^{q_{1}-2}\left(1-\left|\varphi_{a_{m}}(w)\right|^{2}\right)^{s} \mathrm{~d} m(w)=+\infty
$$

Proof. As in [1], we can deduce that $\mathcal{N}_{n} \cap B_{q, q-2, ; n}^{\#}$. Also, we have

(i) $B^{\#}(q, q-2, s ; n) \subset \mathcal{N}_{n}$ for all $q$, with $2<q<\infty$ and $0<s<1$.

$$
\bigcup_{2<q<q_{1}} B^{\#}(q, q-2, s ; n) \subsetneq B^{\#}\left(q_{1}, q_{1}-2, s ; n\right) .
$$

(ii) For all values of $q, q_{1}, 2<q<\infty, 0<s<1$ with Therefore, it is obvious to get that $q_{1}+s>1$, we have that

$$
\lim _{m \rightarrow \infty} \iint_{U}\left[\frac{\left|h^{(n)}(w)\right|}{1+|h(w)|^{n+1}}\right]^{q_{1}}\left(1-|w|^{2}\right)^{q_{1}-2}\left(1-\left|\varphi_{a_{m}}(w)\right|^{2}\right)^{s} \mathrm{~d} m(w)=+\infty
$$

Remark 3. The recent developments of fractional calculus as well as its applications are more essential to complex function spaces with the specific arbitrary fractional order derivatives. For some recent interesting studies on the subject of fractional calculus, we can here refer to [17-19] and others. To the best of our knowledge, a few number of manuscripts researched some certain classes of analytic function spaces by the help of general fractional derivatives (see [20]). For further research work, the following specific interesting question can be considered:

How one can define and study the Besov spaces of general meromorphic functions by using the general fractional derivatives?

\section{Conclusions}

Certain concerned weighted classes of meromorphic function spaces using the general spherical derivatives are studied and discussed in this article. The general Besov-type classes of meromorphic functions as well as the general normal functions are considered intensively and both are compared deeply with each other. For a concerned nonnormal function $h$, the concerned families of points $\left\{a_{m}\right\}$ and $\left\{c_{m}\right\}$, for which

$$
\begin{aligned}
& \lim _{m \rightarrow \infty}\left(1-\left|a_{m}\right|^{2}\right) \frac{\left|h^{(n)}\left(a_{m}\right)\right|}{1+\left|h\left(a_{m}\right)\right|^{n+1}}=+\infty, \quad n \in \mathbb{N}, \\
& \lim _{m \rightarrow \infty} \iint_{U}\left[\frac{\left|h^{(n)}(w)\right|}{1+|h(w)|^{n+1}}\right]^{q}\left(1-|w|^{2}\right)^{q-2}\left(1-\left|\varphi_{a_{m}}(w)\right|^{2}\right)^{s} \mathrm{~d} m(w)=+\infty
\end{aligned}
$$

are introduced and discussed. Several connections between families (sequences) of $b_{(q, n)}$ and $q_{(N, n)}$ type are established. The obtained results improve, extend, and generalize numerous results in [21-23].

Remark 4. Quite recently there are some important enjoyable research studies on hyperbolic function classes (see $[24,25])$. For more interesting research, how we can construct some workable conditions on some hyperbolic-type sequences of points that make guarantee to belong to some specific hyperbolic-type classes?

\section{Data Availability}

No data were applied or considered to support this study.

\section{Conflicts of Interest}

The authors declare that they have no conflicts of interest. 


\section{Acknowledgments}

The first author would like to thank Taif University Researchers concerning the support of project no. TURSP2020/159, Taif University, Saudi Arabia.

\section{References}

[1] J. Rättyä, "On some Complex function spaces and classes," Annales Academiae Scientiarum Fennicae. Series A I. Mathematica. Dissertationes, vol. 124, pp. 1-73, 2001.

[2] C. Batty, A. Gomilko, and Y. Tomilov, "A Besov algebra calculus for generators of operator semigroups and related norm-estimates," Mathematische Annalen, vol. 379, no. 1-2, pp. 23-93, 2021.

[3] S. Dominguez and D. Girela, "Radial growth of the derivatives of analytic functions in Besov spaces," Concrete Operators, vol. 8, no. 1, pp. 1-12, 2021.

[4] S. Dominguez and D. Girela, "A radial integrability result concerning bounded functions in analytic Besov spaces with applications," Results in Mathematics, vol. 75, 2020.

[5] K. Stroethoff, "Besov-type characterisations for the Bloch space," Bulletin of the Australian Mathematical Society, vol. 39, no. 3, pp. 405-420, 1989.

[6] M. Tjani, "Compact composition operators on some mobius invariant banach spaces," Thesis, Michigan State University, Michigan, USA, 1996.

[7] X. Zhu and N. Hu, "Weighted composition operators from Besov Zygmund-type spaces into Zygmund-type spaces," Journal of Function Spaces, vol. 2020, Article ID 2384971, 7 pages, 2020.

[8] A. El-Sayed Ahmed and M. Al-Bogami, "Generalizations on some meromorphic function spaces in the unit disc," Journal of Computational Analysis and Applications, vol. 22, no. 1, pp. 148-160, 2017.

[9] R. Aulaskari and P. Lappan, "Criteria for an analytic function to be Bloch and a harmonic or meromorphic function to be normal, Complex analysis and its applications," Longman Scientific and Technical Harlow, vol. 305, pp. 136-146, 1994.

[10] A. El-Sayed Ahmed and A. Kamal, "-type spaces of analytic and meromorphic functions," Mathematica, vol. 54, no. 77, pp. 26-37, 2012.

[11] L. Bernal-Gonzälez, A. Jung, and J. Müller, "Universality vs. non-normality of families of meromorphic functions," Proceedings of the American Mathematical Society, vol. 149, no. 2, pp. 761-771, 2021.

[12] R. Aulaskari and P. Lappan, "Additive automorphic functions and Bloch functions," Canadian Journal of Mathematics, vol. 46, no. 3, pp. 474-484, 1994.

[13] C. Fang and Y. Xu, "Normal family of meromorphic functions concerning fixed-points," Analysis and Mathematical Physics, vol. 9, no. 1, pp. 197-207, 2019.

[14] S. Makhmutov, "Integral characterizations of Bloch functions," New Zealand Journal of Mathematics, vol. 26, pp. 201-212, 1997.

[15] J. L. Schiff, Normal Families, Springer-Verlay, New York, NY, USA, 1993.

[16] H. Wulan, "On some classes of meromorphic functions, Annales Academiae Scientiarum Fennicae. Series A I. Mathematica," Doctoral dissertations, vol. 116, pp. 1-57, Suomalainen Tiedeakatemia, Helsinki, Finland, 1998.

[17] S. Rashid, M. A. Latif, Z. Hammouch, and Y.-M. Chu, "Fractional integral inequalities for strongly h-preinvex functions for a kth order differentiable functions," Symmetry, vol. 11, no. 12, p. 1448, 2019.

[18] S. Rashid, H. Kalsoom, Z. Hammouch, R. Ashraf, D. Baleanu, and Y.-M. Chu, "New multi-parametrized estimates having pth-order differentiability in fractional calculus for predominating $\hbar$-convex functions in hilbert space," Symmetry, vol. 12 , no. 2 , p. 222,2020 .

[19] S. S. Zhou, S. Rashid, S. Parveen, A. O. Akdemir, and Z. Hammouch, "New computations for extended weighted functionals within the Hilfer generalized proportional fractional integral operators," AIMS Mathematics, vol. 6, no. 5, pp. 4507-4525, 2021.

[20] A. Perälä, "General fractional derivatives and the Bergman projection," Annales Academiae Scientiarum Fennicae Mathematica, vol. 45, no. 2, pp. 903-913, 2020.

[21] A. El-Sayed Ahmed and M. A. Bakhit, "Sequences of some meromorphic function spaces," Bulletin of the Belgian Mathematical Society-Simon Stevin, vol. 16, no. 3, pp. 395408, 2009.

[22] R. Aulaskari, S. Makhmutov, and H. Wulan, "On Q p Sequences," Finite or Infinite Dimensional Complex Analysis and Applications, vol. 2, pp. 117-125, 2004.

[23] R. Aulaskari, S. Makhmutov, and J. Rättyä, "Hyperbolically close to $Q_{p}^{\#}$-sequences," Bulletin of the Korean Mathematical Society, vol. 57, no. 1, pp. 133-138, 2020.

[24] A. El-Sayed Ahmed, "Refinements on some classes of complex function spaces," Symmetry, vol. 13, no. 2, p. 339, 2021.

[25] A. El-Sayed Ahmed and A. E. Shammaky, "Certain classes of operators on some weighted hyperbolic function spaces," Journal of Mathematics, vol. 2021, Article ID 6664398, 6 pages, 2021. 